\title{
Transmission of pillar-based photonic crystal waveguides in InP technology
}

\author{
Abigaël Kok, ${ }^{a)}$ Erik Jan Geluk, Boudewijn Docter, Jos van der Tol, \\ Richard Nötzel, and Meint Smit \\ COBRA Research Institute, Eindhoven University of Technology, P.O. Box 513, 5600 MB Eindhoven, \\ The Netherlands \\ Roel Baets \\ Department of Information Technology, Ghent University-IMEC, Sint-Pietersnieuwstraat 41, \\ 9000 Gent, Belgium
}

(Received 12 October 2007; accepted 29 October 2007; published online 14 November 2007)

\begin{abstract}
Waveguides based on line defects in pillar photonic crystals have been fabricated in $\mathrm{InP} / \mathrm{InGaAsP} / \mathrm{InP}$ technology. Transmission measurements of different line defects are reported. The results can be explained by comparison with two-dimensional band diagram simulations. The losses increase substantially at mode crossings and in the slow light regime. The agreement with the band diagrams implies a good control on the dimensions of the fabricated features, which is an important step in the actual application of these devices in photonic integrated circuits.

(C) 2007 American Institute of Physics. [DOI: 10.1063/1.2814041]
\end{abstract}

Photonic crystal $(\mathrm{PhC})$ slabs offer huge possibilities for devices with small footprints in integrated optical circuits. In these structures, the photonic band gap confines the light in plane, whereas the total internal reflection is exploited for confinement in the vertical dimension. ${ }^{1} \mathrm{PhCs}$ based on pillars have several advantages compared to hole-type PhCs. To start with, the layer stack of pillar PhCs is compatible with that of the classical photonic integrated circuits (PICs), and so is the fabrication technology. Furthermore, active pillarbased devices can be electrically contacted, inherently avoiding current spreading, and heat is efficiently dissipated to the substrate.

In this letter, we describe transmission measurements on pillar-based $\mathrm{PhC}$ waveguides on a double heterostructure consisting of an InP substrate $[n=3.169$ at $\lambda=1550 \mathrm{~nm}$ (Ref. $2)]$, an InGaAsP $[Q(1.25 \mu \mathrm{m})]$ guiding layer of $500 \mathrm{~nm}$ thickness $(n=3.364)$, and an InP top cladding of $1 \mu \mathrm{m}$. The waveguiding structure, as schematically shown in Fig. 1, consists of a 1.8- $\mu \mathrm{m}$-wide input ridge waveguide, followed by a $1 \times 2$ multimode interference (MMI) coupler splitting the light into two branches. In the reference branch, the light propagates through a classical ridge waveguide toward the output side of the chip. The other branch contains a $\mathrm{PhC}$ waveguide with a length of 16 periods. This configuration has two major advantages; first, the incoupling of light into the input waveguide can easily be optimized using the reference branch, even if the $\mathrm{PhC}$ waveguide has high losses, and second, the transmission of the $\mathrm{PhC}$ waveguide can directly be calculated from a comparison with the reference arm.

$\mathrm{A} \mathrm{PhC} \mathrm{consisting} \mathrm{of} \mathrm{a} \mathrm{square} \mathrm{lattice} \mathrm{of} \mathrm{high-index} \mathrm{pillars}$ exhibits a large TM band gap, where TM is the polarization with the electric field vector parallel to the axes of the pillars. ${ }^{3}$ This band gap is exploited to create a waveguide by introducing a line defect of larger pillars. Since we aim for operation in PICs in the wavelength range from 1530 to $1570 \mathrm{~nm}$, the $\mathrm{PhC}$ waveguides are designed for transmission at around $\lambda=1550 \mathrm{~nm}$. A large photonic band

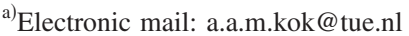

gap is obtained for a pillar $\mathrm{PhC}$ with a lattice constant $a$ $=491 \mathrm{~nm}$ and a radius $r=123 \mathrm{~nm}$. Figure 2 shows the calculated two-dimensional (2D) band diagrams of line defects with varying radius of the defect pillars $\left(r_{\text {defect }}\right)$. To account for the third dimension, the effective refractive index of the pillars is used. The waveguide mode with a monotonically decreasing dispersion curve has an even mode profile, whereas the mode with the increasing dispersion curve has an odd symmetry. For increasing defect pillar radius, the frequencies of both the modes are reduced due to the higher average refractive index of the line defect.

Light is coupled from the ridge waveguide to the $\mathrm{PhC}$ waveguide by first adiabatically tapering the ridge waveguide down to a width that matches the diameter of the defect pillars. The ridge waveguide is then placed in front of the $\mathrm{PhC}$ waveguide, ${ }^{4}$ with a spacing that equals half of the distance between two adjacent defect pillars [i.e., a spacing of $\left(a-2 r_{\text {defect }}\right) / 2$ between the end facet of the ridge and the first waveguide pillar], which should give the highest transmission according to simulations. Since the single-mode ridge waveguide is placed symmetrically in front of the photonic crystal waveguide, we expect to excite mainly the guided mode with even symmetry.

The ridge waveguides and the MMIs are defined by optical lithography, whereas the $\mathrm{PhC}$ waveguides are defined by electron beam lithography. At the transition between the optically defined waveguides and the e-beam lithography ar-

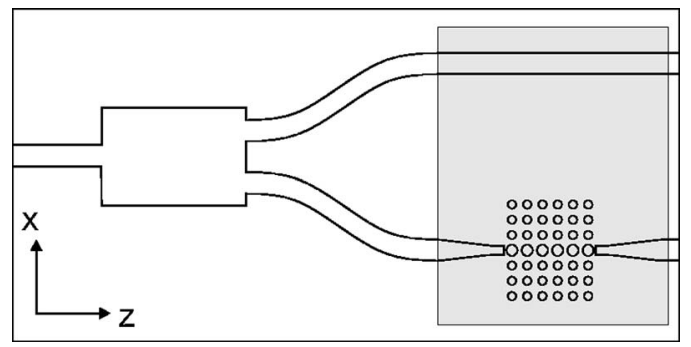

FIG. 1. Schematic drawing of the chip layout, where the gray area is defined by e-beam lithography. 


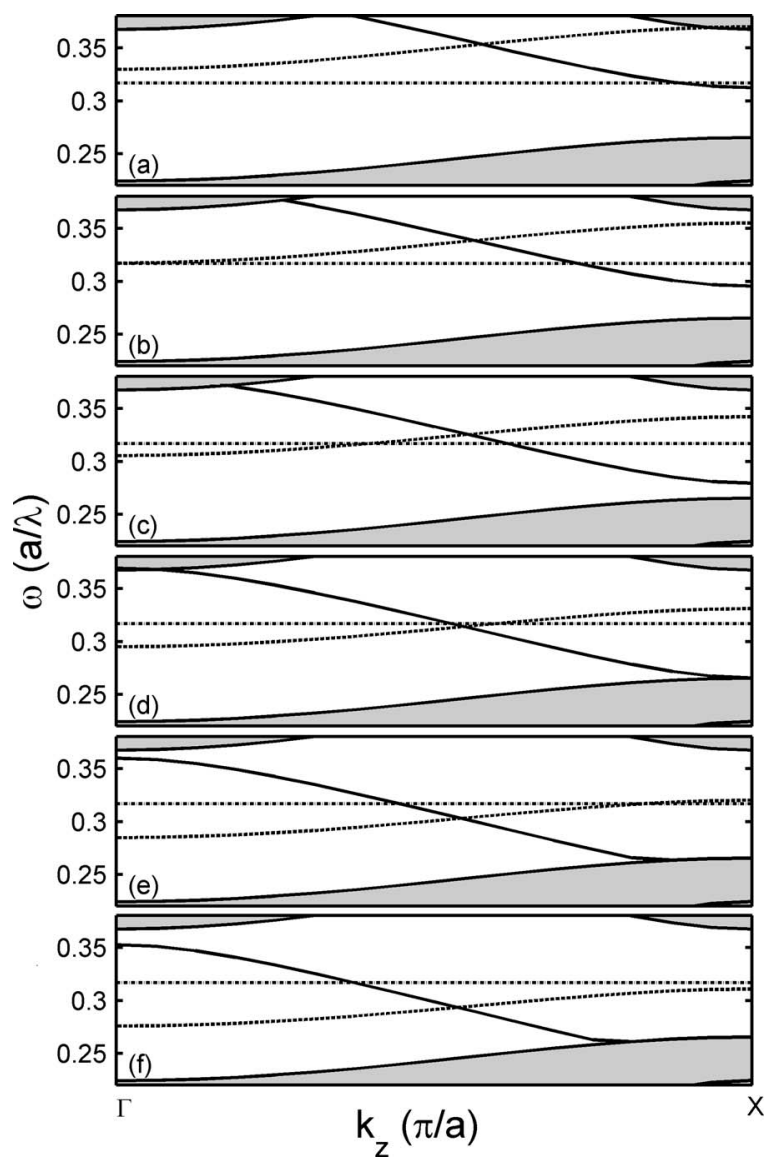

FIG. 2. Comparison of band diagrams for varying defect pillar radius, $r_{\text {defect }}$ equals (a) $160 \mathrm{~nm}$, (b) $170 \mathrm{~nm}$, (c) $180 \mathrm{~nm}$, (d) $190 \mathrm{~nm}$, (e) $200 \mathrm{~nm}$, and (f) $210 \mathrm{~nm}$. The gray areas indicate the PhC modes supported by the surrounding photonic crystal. The solid lines belong to the even waveguide mode, the dashed line to the odd waveguide mode, and the dash-dotted line represents the frequency corresponding to $\lambda=1550 \mathrm{~nm}$.

eas, the waveguides are $0.8 \mu \mathrm{m}$ wide. The waveguide pattern, including the photonic crystals, is first defined in a 50-nm-thick chromium masking layer using a lift-off process. This pattern is transferred into a 430-nm-thick silicon dioxide layer by reactive ion etching (RIE) using a $\mathrm{CHF}_{3}$ chemistry. Finally, the deep etch to create the waveguides is performed by inductively coupled plasma (ICP) RIE using a $\mathrm{Cl}_{2}: \mathrm{Ar}: \mathrm{H}_{2}$ chemistry. ${ }^{5}$ Figure 3 shows a scanning electron microscopy (SEM) image of the $\mathrm{PhC}$ waveguide after the

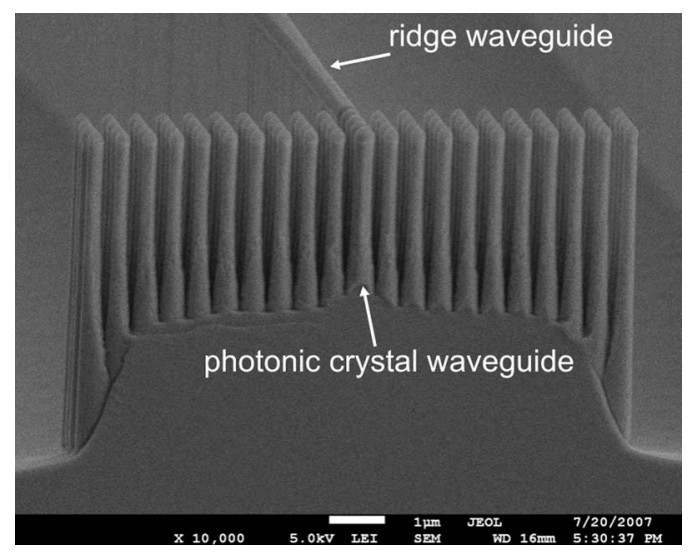

FIG. 3. SEM image of a cross section of a $\mathrm{PhC}$ waveguide connected to a classical ridge waveguide on an InP substrate. In the characterized devices the silicon dioxide masking layer has been removed.

Downloaded 17 Jan 2008 to 157.193.172.109. Redistribution subject to AIP license or copyright; see http://apl.aip.org/apl/copyright.jsp

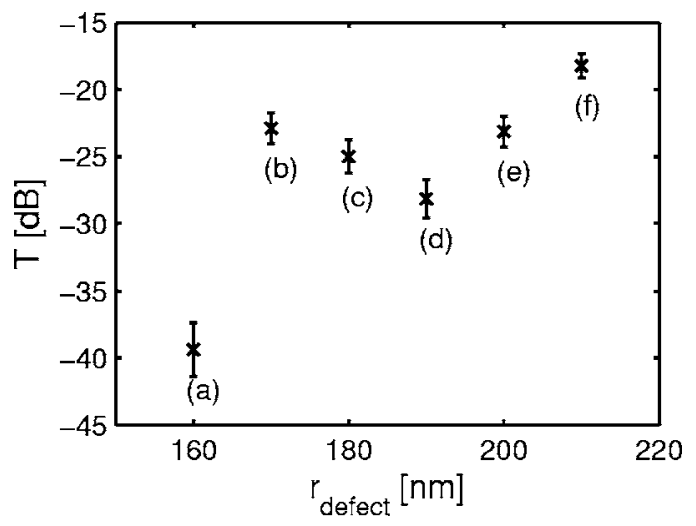

FIG. 4. Measured transmission as a function of the radius of the defect Fig. 2.

ICP etch. The pillars are $\sim 3.0 \mu \mathrm{m}$ deep. According to simulations, this should be enough to minimize coupling to the substrate modes. ${ }^{6}$

TM-polarized light from a tunable laser source is coupled into the input waveguide using a microscope objective. At the output side, the transmitted light is collected with a lensed fiber and measured by a photoreceiver. The tunable laser scans the wavelength from 1530 to $1570 \mathrm{~nm}$ in steps of $0.1 \mathrm{~nm}$. The cleaved facets of the chip introduce Fabry-Pérot fringes on the measured spectrum. These are averaged out by taking a running average over ten data points of the spectrum. From the averaged spectra of both the branches, the transmission of the photonic crystal waveguide is determined. The transmission losses include the tapering of a waveguide from $0.8 \mu \mathrm{m}$ width down to the diameter of the defect pillars, the coupling to and from the $\mathrm{PhC}$ waveguide, and the propagation loss in the $\mathrm{PhC}$ waveguide.

Figure 4 shows the measured transmission through a 16period-long $\mathrm{PhC}$ waveguide as a function of the defect pillar radius. The transmission of each $\mathrm{PhC}$ waveguide is averaged over the scanned wavelength range, and the bars indicate the standard deviation from the mean value over this wavelength range. The dependence of the loss on the defect pillar radius cannot be explained by only diffraction effects, since in that case the transmission would monotonically increase with the defect radius. However, it can be understood by comparison with the in-plane propagation constants $k_{z}$ and the group velocities $v_{\mathrm{g}}$ of the guided modes. For convenience, the calculated group velocities of the guided modes are plotted in Fig. 5 . Note that the even mode has a higher group velocity than the odd mode. If the group velocity is low, the light propagates relatively slowly through the waveguide, resulting in longer interaction times with the crystal and therefore higher losses.

For a defect radius as small as $r_{\text {defect }}=160 \mathrm{~nm}$, the even mode is the only guided mode [see Fig. 2(a)], but this mode approaches the slow light regime and therefore exhibits the lowest transmission. Also, the mode is very close to cutoff, and if the defect radius is only $5 \mathrm{~nm}$ smaller in the realized device, no guided mode is present and the $\mathrm{PhC}$ has a band gap at $\lambda=1550 \mathrm{~nm}$. For a defect radius of $170 \mathrm{~nm}$, as shown in Figs. 2(b) and 5, the odd mode is absent at $\lambda=1550 \mathrm{~nm}$ and the even mode has a high group velocity. This results in relatively low $\mathrm{PhC}$ waveguide losses. Increasing the defect radius to $180 \mathrm{~nm}$ introduces the odd mode at $\lambda=1550 \mathrm{~nm}$ pillars. The notations (a) to (f) refer to the corresponding band diagrams of 


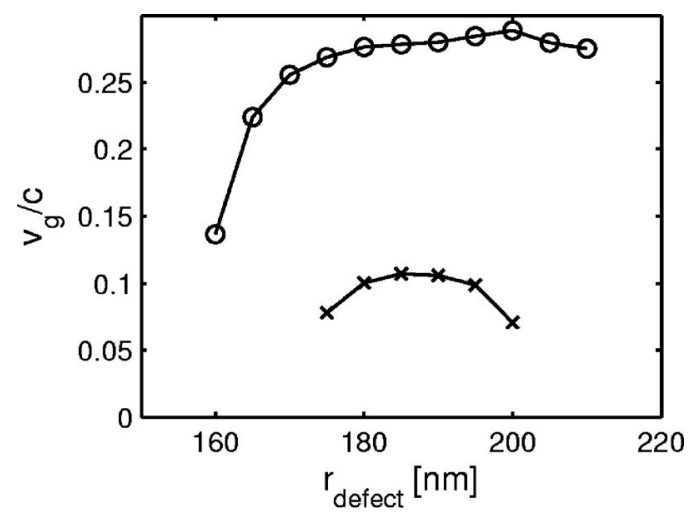

FIG. 5. Group velocities of both the even mode (circles) and the odd mode (crosses) as a function of the defect pillar radius at $\lambda=1550 \mathrm{~nm}$.

[see Fig. 2(c)], which might be excited if the symmetry of the structure is broken, e.g., by fabrication imperfections. Apart from the lower group velocity, this mode is not matched to the output ridge waveguide, and it will exhibit high coupling losses. Indeed, the measured losses are higher than those of a waveguide with $r_{\text {defect }}=170 \mathrm{~nm}$.

For a waveguide with $r_{\text {defect }}=190 \mathrm{~nm}$, the crossing of the even and the odd modes is close to the frequency corresponding to $\lambda=1550 \mathrm{~nm}$. The $\boldsymbol{k}_{z}$ vectors of both modes are nearly equal, and light can easily couple from one mode to the other (e.g., induced by fabrication imperfections), resulting in even higher loss. Increasing the defect radius to $r_{\text {defect }}=200 \mathrm{~nm}$ increases the difference between the $\boldsymbol{k}_{z}$ vectors of the modes and the situation is comparable to that of $r_{\text {defect }}=180 \mathrm{~nm}$. Finally, for $r_{\text {defect }}=210 \mathrm{~nm}$, the odd mode is cutoff and the even mode has a high group velocity. This is the $\mathrm{PhC}$ waveguide with the highest transmission.

In summary, the measured transmission of a $\mathrm{PhC}$ waveguide based on pillars is in good agreement with the 2D band diagram simulations of the line defects. This implies that the fabrication technology can be accurately controlled, which is crucial if more complicated $\mathrm{PhC}$ components are designed. The highest transmission is obtained for PhCs where mode crossings and slow light propagation are avoided. The losses are high, but they can be reduced by implementation of a guiding layer stack between the pillars. ${ }^{7}$ By developing a technology platform for pillar PhCs, a huge number of new applications in classical PICs is within reach.

This research is supported by NanoNed, a technology programe of the Dutch Ministry of Economic Affairs.

${ }^{1}$ S. G. Johnson, S. Fan, P. R. Villeneuve, and J. D. Joannopoulos, Phys. Rev. B 60, 5751 (1999).

${ }^{2}$ GaInAsP Alloy Semiconductors, edited by T. D. Pearsall (Wiley, New York, 1982).

${ }^{3}$ J. D. Joannopoulos, R. D. Meade, and J. N. Winn, Photonic Crystals (Princeton University Press, Princeton, 1995).

${ }^{4}$ A. A. M. Kok, R. Meneghelli, J. J. G. M. van der Tol, and M. K. Smit, Proceedings of the IEEE/LEOS Benelux Symposium, The Netherlands, 2006 (unpublished), p. 37.

${ }^{5}$ B. Docter, E. J. Geluk, M. J. H. Sander-Jochem, F. Karouta, and M. K. Smit, Proceedings of the IPRM, Japan, 2007 (unpublished), p. 226.

${ }^{6}$ A. A. M. Kok, J. J. G. M. van der Tol, R. Baets, and M. K. Smit, "Reduction of propagation loss in pillar-based photonic crystal waveguides," J. Lightwave Technol. (to be published).

${ }^{7}$ A. A. M. Kok, E. J. Geluk, M. J. H. Sander-Jochem, J. J. G. M. van der Tol, Y. S. Oei, and M. K. Smit, Proceedings of the IEEE/LEOS Benelux Symposium Belgium, 2005 (unpublished), p. 273. 